\title{
Factor Structure of the Targeted Inventory on Problems in Schizophrenia
}

\author{
Shoji Tanaka1, Takanori Nagase ${ }^{1}$, Takefumi Suzuki ${ }^{2}$, Kensuke Nomura ${ }^{2}$, Hiroyoshi Takeuchi $^{2}$, Shinichiro \\ Nakajima ${ }^{2}$, Hiroyuki Uchida ${ }^{2}$, Gohei Yagi $^{2}$, Koichiro Watanabe ${ }^{2}$, Masaru Mimura ${ }^{2}$ \\ ${ }^{1}$ Department of Information and Communication Sciences, Sophia University, ${ }^{2}$ Department of Neuropsychiatry, Keio University School of \\ Medicine, Tokyo, Japan
}

\begin{abstract}
Objective: The aim of this study was to explore the factor structure of a novel, 10-item rating scale, the Targeted Inventory on Problems in Schizophrenia (TIP-Sz). Determining the factor structure will be useful in the brief evaluation of medication and non-medication treatment of the disease.

Methods: An exploratory factor analysis was performed on TIP-Sz scores obtained from 100 patients who met the Diagnostic and Statistical Manual of Mental Disorders (DSM-IV) criteria for schizophrenia,

Results: The factor analysis extracted four factors that were deemed clinically pertinent, which we labeled: disorganization, social cooperativeness, functional capacity, and emotional state. The items exhibited cross-loadings on the first three factors (i.e., some items loaded on more than one factor). In particular, the 'behavioral dyscontrol and disorganization,' 'insight and reality testing,' and 'overall prognostic impression' items had comparable cross-loadings on all of the first three factors. The emotional state factor was distinct from the other factors in that the items loading on it did not cross-load on other factors.

Conclusion: The TIP-Sz scale comprises factors that are associated with the psychosocial functioning and emotional state of patients, which are important outcome parameters for successful treatment of the disease.
\end{abstract}

KEY WORDS: Disorganization; Negative symptom; Positive symptom; Rating scale; Social functioning.

\section{INTRODUCTION}

Recently, a novel assessment scale, the Targeted Inventory on Problems in Schizophrenia (TIP-Sz), was created in an effort to facilitate measurement-based treatment of schizophrenia. ${ }^{1)}$ This scale includes important aspects of the disease, such as adherence to treatment, therapeutic alliance, comfort of the therapist with the situation, subjective well-being, and satisfaction with therapy. This scale consists of 10 items that address highly problematic issues in the clinical management of patients with schizophrenia that are commonly encountered in the real world. TIP-Sz 1 assesses the degree of loss of self-control and organization during conversation and in behavior. TIP-Sz 2 assesses hostility, agitation, and violence, regardless of whether patients are psychotic.

\footnotetext{
Received: July 9, 2012 / Revised: September 12, 2012

Accepted: September 28, 2012

Address for correspondence: Shoji Tanaka, PhD

Department of Information and Communication Sciences,

Sophia University, 7-1 Kioicho, Chiyoda-ku, Tokyo 102-8554, Japan

Tel: +81-3-3238-3331, Fax: +81-3-3238-3321

E-mail: tanaka-s@sophia.ac.jp
}

TIP-Sz 3 assesses indifference, affective withdrawal, and motor retardation. TIP-Sz 4 assesses symptoms of mood, anxiety, obsession, and compulsion. TIP-Sz 5 evaluates patients' insight into their illness, their understanding of their past, present, and future, and their thought content from the viewpoint of societal norms regarding acceptability and deviance. TIP-Sz 6 assesses social competence, independence, and self-care capability including appearance, communication, and working skills. TIP-Sz 7 assesses adherence to therapy, which is one of the most important determinants of successful treatment. TIP-Sz 8 assesses the therapeutic alliance and comfort of the therapist with the situation. This item evaluates the patient from the therapist's point of view. TIP-Sz 9 taps the overall prognostic impression based on all available information about the patient and the best knowledge of the rater. TIP-Sz 10 assesses subjective well-being and satisfaction with therapy. This item aims to provide a simple representation of subjective quality of life. For a complete description of the items, the reader is referred to the original publication. ${ }^{1)}$

Exploratory factor analysis has been used to delineate the meaningful dimensions of schizophrenia symptoms.

(c) This is an Open-Access article distributed under the terms of the Creative Commons Attribution Non-Commercial License (http://creativecommons.org/licenses/by-nc/3.0) which permits unrestricted non-commercial use, distribution, and reproduction in any medium, provided the original work is properly cited. 
Departing from the positive-negative dichotomization of schizophrenia symptoms, ${ }^{2,3)}$ a number of studies have conducted dimensional analyses of schizophrenia symptoms based on the established Scale for the Assessment of Positive Symptoms (SAPS) and the Scale for the Assessment of Negative Symptoms (SANS). These studies have generally yielded converging results, indicating that the symptoms can be explained mainly by three factors: positive symptoms, negative symptoms, and disorganization. ${ }^{4)}$ A later factor analysis of all 50 items in the SAPS and SANS extracted five factors: diminished expression, disorganization, disordered relating, bizarre delusions, and auditory hallucinations. ${ }^{5)}$ The 30-item Positive and Negative Syndrome Scale (PANSS) originally consisted of three subscales: positive symptoms, negative symptoms, and general psychopathology. ${ }^{6}$ A number of studies subsequently performed factor analyses and principal component analyses on PANSS scores. Although the details differ, most of the studies generally converged on a five-factor model: positive symptoms, negative symptoms, disorganization, excitement, and anxiety/depression. ${ }^{7-15)} \mathrm{A}$ subsequent analysis with a larger sample size $(n=5,769)$ supported the five-factor model of the PANSS. ${ }^{16,17)}$ Furthermore, the five-factor model of the PANSS appears to be robust across various phases of the illness and is suggested to remain stable after antipsychotic treatment. ${ }^{18)}$ Thus, this model is often used in the analysis of symptoms and medication effects in schizophrenia.

The number of the TIP-Sz items is rather small, so that one can complete TIP-Sz scoring in a much shorter time as compared with PANSS scoring. The aim of this study was to clarify the structure of the rating scale to advance the understanding of the disease symptoms. A factor model extracted from TIP-Sz scores would be useful in evaluating treatment effects, as it would be possible to evaluate treatment effects by changes in factor scores rather than changes in the scores on individual items. Factor scores are more general than individual item scores, so the evaluation is compatible across different rating scales to some extent. In this article, we first present factor analysis results of the TIP-Sz based on scores obtained from patients with schizophrenia. Second, we discuss the plausibility of the extracted factor model.

\section{METHODS}

\section{Participants}

The participants were outpatients and inpatients who met the Diagnostic and Statistical Manual of Mental
Disorders (DSM-IV) criteria for schizophrenia. One hundred patients ( 58 males and 42 females; 40 outpatients and 60 inpatients) participated. The data comprised two independent data sets. The first data set was used in a previous study. ${ }^{1)}$ That study included 36 subjects (18 males and 18 females; 18 inpatients and 18 outpatients). The mean age was 47.6 years (standard deviation $[\mathrm{SD}]=14.7$; range, 23-74 years). The mean scores were as follows: PANSS total (mean=108.5, $\mathrm{SD}=19.4$ for the inpatients; mean $=82.3, \mathrm{SD}=15.0$ for the outpatients) and TIP-Sz total (mean=49.5, $\mathrm{SD}=9.8$ for the inpatients; mean=71.4, $\mathrm{SD}=8.2$ for the outpatients). The second data set consisted of data collected from 64 other patients with schizophrenia sampled from the same institution in 2011 (40 male and 24 female; 42 inpatients and 22 outpatients). The mean age was 45.7 years ( $\mathrm{SD}=13.1$; range, 21-69 years). We did not have PANSS scores for these participants. The mean TIP-Sz scores were $40.5(\mathrm{SD}=16.1)$ for the inpatients and $70.6(\mathrm{SD}=8.6)$ for the outpatients. This study and the previous study ${ }^{1)}$ received ethical approval from the participating sites. Written informed consent was obtained from all of the participants in the original investigation, and the requirement for additional informed consent was waived because the study data were handled anonymously. At the time of the second sampling, written informed consent was obtained from all of the participants after receiving ethical approval from the participating site.

\section{TIP-Sz}

The TIP-Sz consists of 10 items and is intended to briefly evaluate problems that are commonly encountered by patients with schizophrenia. The score on each item ranges from zero to 10 , with higher scores indicating a less severe clinical status. A detailed description of the TIP-Sz was provided in a previous paper. ${ }^{1)}$

\section{Analysis}

An exploratory factor analysis with varimax rotation was performed on the TIP-Sz scores to extract independent factors. We ran the entire analysis using $\mathrm{R}$ ( $\mathrm{R}$ Foundation for Statistical Computing, Vienna, Austria).

\section{RESULTS}

Pearson's correlations between the TIP-Sz scores are shown in Table 1. The fifth item in the TIP-Sz (insight and reality testing) was strongly correlated with many other items, including TIP-Sz 1 (behavioral dyscontrol and disorganization), TIP-Sz 6 (social competence and in- 
Table 1. Pearson's Correlation Matrix of the TIP-Sz Scores

\begin{tabular}{|c|c|c|c|c|c|c|c|c|c|c|}
\hline & TIP-Sz 1 & TIP-Sz 2 & TIP-Sz 3 & TIP-Sz 4 & TIP-Sz 5 & TIP-Sz 6 & TIP-Sz 7 & TIP-Sz 8 & TIP-Sz 9 & TIP-Sz 10 \\
\hline TIP-Sz 1 & 1 & & & & & & & & & \\
\hline TIP-Sz 2 & 0.568 & 1 & & & & & & & & \\
\hline TIP-Sz 3 & 0.561 & 0.47 & 1 & & & & & & & \\
\hline TIP-Sz 4 & 0.218 & 0.393 & 0.224 & 1 & & & & & & \\
\hline TIP-Sz 5 & 0.764 & 0.633 & 0.661 & 0.124 & 1 & & & & & \\
\hline TIP-Sz 6 & 0.679 & 0.621 & 0.831 & 0.330 & 0.776 & 1 & & & & \\
\hline TIP-Sz 7 & 0.583 & 0.600 & 0.643 & 0.263 & 0.718 & 0.725 & 1 & & & \\
\hline TIP-Sz 8 & 0.615 & 0.758 & 0.575 & 0.367 & 0.748 & 0.698 & 0.731 & 1 & & \\
\hline TIP-Sz 9 & 0.770 & 0.596 & 0.780 & 0.284 & 0.848 & 0.861 & 0.718 & 0.741 & 1 & \\
\hline TIP-Sz 10 & 0.089 & 0.191 & 0.132 & 0.423 & 0.040 & 0.162 & 0.217 & 0.213 & 0.136 & 1 \\
\hline
\end{tabular}

The correlations that were greater than 0.700 (with the exception of the diagonal elements) are indicated in bold type.

TIP-Sz, the Targeted Inventory on Problems in Schizophrenia.

Table 2. Factor Loadings of the TIP-Sz Items

\begin{tabular}{|c|c|c|c|c|}
\hline & $\begin{array}{l}\text { Disorgani- } \\
\text { zation }\end{array}$ & $\begin{array}{c}\text { Social } \\
\text { cooperativeness }\end{array}$ & $\begin{array}{l}\text { Functional } \\
\text { capacity }\end{array}$ & $\begin{array}{l}\text { Emotional } \\
\text { state }\end{array}$ \\
\hline TIP-Sz 1 & 0.618 & 0.411 & 0.415 & \\
\hline TIP-Sz 2 & & 0.673 & & \\
\hline TIP-Sz 3 & & & 0.870 & \\
\hline TIP-Sz 4 & & & & 0.898 \\
\hline TIP-Sz 5 & 0.504 & 0.596 & 0.516 & \\
\hline TIP-Sz 6 & & 0.402 & 0.760 & \\
\hline TIP-Sz 7 & & 0.585 & 0.518 & \\
\hline TIP-Sz 8 & & 0.803 & & \\
\hline TIP-Sz 9 & 0.476 & 0.453 & 0.670 & \\
\hline TIP-Sz 10 & & & & 0.458 \\
\hline
\end{tabular}

The factor analysis used varimax rotation. The cumulative variance is $75.1 \%$; the proportion variance of each factor was $27.0 \%$ (functional capacity), $24.2 \%$ (social cooperativeness) $12.7 \%$ (emotional state), and $11.3 \%$ (disorganization). Only factor loadings greater than 0.400 are shown. The highest factor loading for each item is indicated in bold type.

TIP-Sz, the Targeted Inventory on Problems in Schizophrenia.

dependence), TIP-Sz 7 (adherence to treatment), TIP-Sz 8 (therapeutic alliance and the therapist's comfort with the situation), and TIP-Sz 9 (overall prognostic impression). However, TIP-Sz 4 (symptoms involving mood, anxiety, obsessions, and compulsions) and TIP-Sz 10 (subjective well-being and satisfaction with therapy) were not strongly correlated with any other items.

The exploratory factor analysis extracted four factors (Table 2 and Fig. 1). Because TIP-Sz 1 had the highest loading on the first factor, this factor was labeled disorganization. TIP-Sz 2 (hostility, agitation and violence), 5, 7, and 8 had the highest loadings on the second factor; therefore, this factor was labeled social cooperativeness. TIP-Sz 3 (indifference, affective withdrawal and motor retardation), 6 , and 9 had the highest loadings on the third factor, which was labeled functional capacity. TIP-Sz 4 and 10 had the highest loadings on the fourth factor, which was labeled emotional state. These four factors explained $75.1 \%$ of the total variance; the proportion var- iance for each factor was $27.0 \%$ (functional capacity), $24.2 \%$ (social cooperativeness), $12.7 \%$ (emotional state), and $11.3 \%$ (disorganization). The uniqueness of the TIP-Sz items was as follows: 0.273 (TIP-Sz 1), 0.340 (TIP-Sz 2), 0.144 (TIP-Sz 3), 0.155 (TIP-Sz 4), 0.121 (TIP-Sz 5), 0.126 (TIP-Sz 6), 0.328 (TIP-Sz 7), 0.124 (TIP-Sz 8), 0.104 (TIP-Sz 9), and 0.773 (TIP-Sz 10). TIP-Sz 4 and TIP-Sz 10 were only weakly correlated with the remaining items, as noted above, and TIP-Sz 10 was the only item with robust uniqueness.

\section{DISCUSSION}

The chief aim of this study was to reveal the factor structure of the TIP-Sz rating scale. An exploratory factor analysis extracted four independent factors that were deemed clinically pertinent: disorganization, social cooperativeness, functional capacity, and emotional state. Our result showed that all items except TIP-Sz 4 and 10 had cross-loadings on the first three factors, indicating the somewhat overlapping nature of these factors. In contrast to the first three factors, the emotional state factor was found to be distinct in that none of the items loading on it cross-loaded on the other factors. The isolation of the emotional state factor was preserved even in the two-factor model that was extracted previously from the data obtained from a smaller sample size $(\mathrm{n}=36){ }^{1)}$ Those two factors were labeled disorganization, social cooperativeness, and functional capacity and emotional state. The emotional state factor was identical to that found in the present four-factor model. The two-factor model stressed the uniqueness of the emotional state factor. The correlation analysis (Table 2) showed that TIP-Sz 4 and 10 were weakly correlated with the other items (all of the correlation coefficients were less than 0.400), whereas the correlation coefficient between TIP-Sz 4 and 10 was 0.423 , 


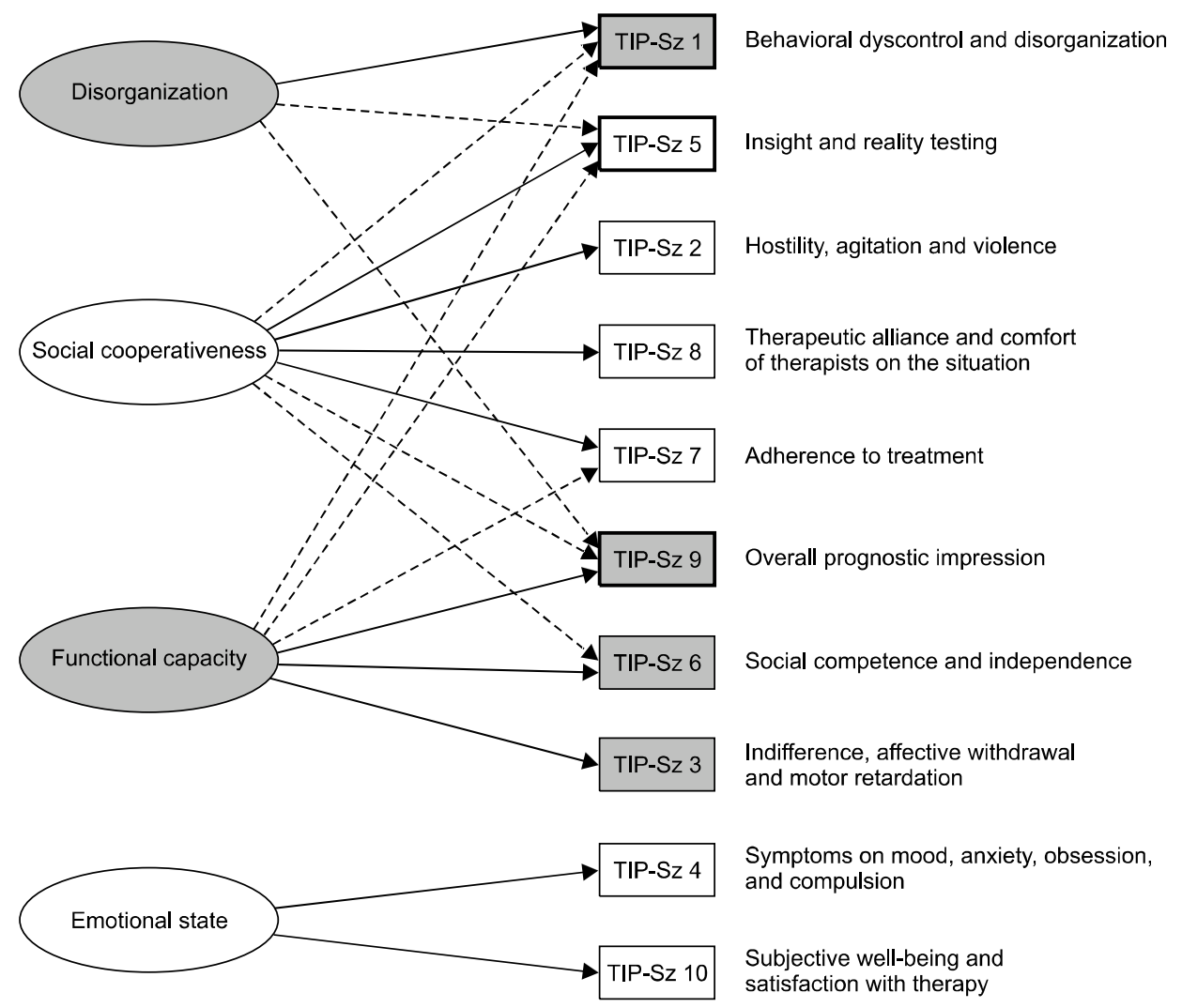

Fig. 1. Factor structure of the TIP-Sz. The four factors extracted from the exploratory factor analysis are shown in ovals, whereas the ten items in the TIP-Sz are shown in rectangles. TIP-Sz 1, TIP-Sz 5, and TIP-Sz 9 have multiple cross-loadings onto the three factors: disorganization, social cooperativeness, and functional capacity. TIP-Sz, the Targeted Inventory on Problems in Schizophrenia.

supporting the uniqueness of this factor. Because the evaluation of these items depends on subjective judgment, a subscale consisting of items 4 and 10 would be important for assessing the subjective feeling of difficulties that patients may have.

The other factor in the two-factor model became separated into three factors (i.e., disorganization, social cooperativeness, and functional capacity) in the present four-factor model. Among the three factors in the four-factor model, the social cooperativeness factor included seven of 10 items (TIP-Sz 1, 2, 5, 6, 7, 8, and 9). In particular, TIP-Sz 2 and 8 (hostility, agitation, and violence and the therapeutic alliance and therapist's comfort with the situation, respectively) exclusively loaded on this factor. This finding suggests that TIP-Sz scores are sensitive to social cooperativeness, which is at times impaired in patients with schizophrenia. ${ }^{19)}$ The widespread influence of this factor is consistent with the previous notion that psychosocial functioning is associated with cognitive difficulty and a range of other clinical symptoms of schizophrenia. $^{20-24)}$
The emotional state factor and the remaining three factors of the TIP-Sz scale were associated with the quality of life and psychosocial functioning of patients, respectively, which are important outcome parameters. Because successful treatment of patients should aim to improve these parameters rather than merely focus on symptom reduction, ${ }^{25)}$ the TIP-Sz factor model would be useful in evaluating treatment effects. Symptom analysis and the evaluation of medication effects using factors other than individual items in a rating scale would be beneficial. First, the number of factors (usually three to five) is usually much smaller than the number of items in the rating scale; therefore, factor models can readily characterize disease symptoms and medication effects. Second, factors would be compatible with the description of symptoms at the phenotypic level so that even if individual items differ between rating scales, factors extracted from those scales may be consistent across scales. This is advantageous for comparison of studies with different rating scales. Third, a factor model facilitates the advancement of a dimensional approach. ${ }^{26)}$ A dimensional approach with factor analysis 
is also capable of finding a new dimension of a disease. ${ }^{27)}$ Multidimensional analysis of schizophrenia symptoms is necessary for deeper understanding of the disease and is also useful in comorbidity studies. ${ }^{28,29)}$

Our exploratory factor analysis extracted a four-factor model of the TIP-Sz. The factors were disorganization, social cooperativeness, functional capacity, and emotional state. Most items exhibited cross-loadings on the first three factors, suggesting that some of the items are associated with more than one factor. In particular, the items addressing behavioral dyscontrol and disorganization, insight and reality testing, and overall prognostic impression had comparable cross-loadings on the first three factors. In contrast, the emotional state factor, which addresses patients' subjective emotional difficulties, was distinct from the other factors (i.e., the items loading on this factor did not cross-load on the other factors). Taken together, these results demonstrate that the TIP-Sz scale is composed of factors that are associated with the psychosocial functioning and emotional state of patients, which are important outcome parameters for the successful treatment of the disease.

\section{Acknowledgments}

This study was supported by the Human Informatics Research Center, Sophia University.

\section{REFERENCES}

1. Suzuki T, Uchida H, Nomura $K$, Takeuchi H, Nakajima $S$, Tanabe A, et al. Novel rating scales for schizophrenia Targeted Inventory on Problems in Schizophrenia (TIP-Sz) and Functional Assessment for Comprehensive Treatment of Schizophrenia (FACT-Sz). Schizophr Res 2008;106:328-336.

2. Liddle PF. The symptoms of chronic schizophrenia. A reexamination of the positive-negative dichotomy. $\mathrm{Br} \mathrm{J}$ Psychiatry 1987; 151:145-151.

3. Crow TJ. Molecular pathology of schizophrenia: more than one disease process? Br Med J 1980;280:66-68.

4. Andreasen NC, Nopoulos P, Schultz S, Miller D, Gupta S, Swayze V, et al. Positive and negative symptoms of schizophrenia: past, present, and future. Acta Psychiatr Scand Suppl 1994;384:51-59.

5. Toomey R, Kremen WS, Simpson JC, Samson JA, Seidman LJ, Lyons MJ, et al. Revisiting the factor structure for positive and negative symptoms: evidence from a large heterogeneous group of psychiatric patients. Am J Psychiatry 1997;154:371-377.

6. Kay SR, Fiszbein A, Opler LA. The positive and negative syndrome scale (PANSS) for schizophrenia. Schizophr Bull 1987; 13:261-276.

7. Peralta V, de Leon J, Cuesta MJ. Are there more than two syndromes in schizophrenia? A critique of the positivenegative dichotomy. Br J Psychiatry 1992;161:335-343.

8. Bell MD, Lysaker PH, Beam-Goulet JL, Milstein RM, Lindenmayer JP. Five-component model of schizophrenia: assessing the factorial invariance of the positive and ne- gative syndrome scale. Psychiatry Res 1994;52:295-303.

9. Bell MD, Lysaker PH, Milstein RM, Beam-Goulet JL. Concurrent validity of the cognitive component of schizophrenia: relationship of PANSS scores to neuropsychological assessments. Psychiatry Res 1994;54:51-58.

10. Lindenmayer JP, Grochowski S, Hyman RB. Five factor model of schizophrenia: replication across samples. Schizophr Res 1995;14:229-234.

11. White L, Harvey PD, Opler L, Lindenmayer JP. Empirical assessment of the factorial structure of clinical symptoms in schizophrenia. A multisite, multimodel evaluation of the factorial structure of the Positive and Negative Syndrome Scale. The PANSS Study Group. Psychopathology 1997;30:263274.

12. Nakaya M, Suwa H, Ohmori K. Latent structures underlying schizophrenic symptoms: a five-dimensional model. Schizophr Res 1999;39:39-50.

13. Lykouras L, Oulis P, Psarros K, Daskalopoulou E, Botsis A, Christodoulou GN, et al. Five-factor model of schizophrenic psychopathology: how valid is it? Eur Arch Psychiatry Clin Neurosci 2000;250:93-100.

14. Drake RJ, Dunn G, Tarrier N, Haddock G, Haley C, Lewis S. The evolution of symptoms in the early course of nonaffective psychosis. Schizophr Res 2003;63:171-179.

15. Emsley R, Rabinowitz J, Torreman M; RIS-INT-35 Early Psychosis Global Working Group. The factor structure for the Positive and Negative Syndrome Scale (PANSS) in recent-onset psychosis. Schizophr Res 2003;61:47-57.

16. van der Gaag M, Cuijpers A, Hoffman T, Remijsen M, Hijman R, de Haan L, et al. The five-factor model of the Positive and Negative Syndrome Scale I: confirmatory factor analysis fails to confirm 25 published five-factor solutions. Schizophr Res 2006;85:273-279.

17. van der Gaag M, Hoffman T, Remijsen M, Hijman R, de Haan L, van Meijel B, et al. The five-factor model of the Positive and Negative Syndrome Scale II: a ten-fold crossvalidation of a revised model. Schizophr Res 2006;85:280287.

18. Lindenmayer JP, Harvey PD, Khan A, Kirkpatrick B. Schizophrenia: measurements of psychopathology. Psychiatr Clin North Am 2007;30:339-363.

19. Mueser KT, Tarrier N. Handbook of social functioning in schizophrenia. 1st ed. Boston:Allyn and Bacon;1998.

20. Simonsen C, Sundet K, Vaskinn A, Ueland T, Romm KL, Hellvin T, et al. Psychosocial function in schizophrenia and bipolar disorder: Relationship to neurocognition and clinical symptoms. J Int Neuropsychol Soc 2010;16:771-783.

21. Addington J, Girard TA, Christensen BK, Addington D. Social cognition mediates illness-related and cognitive influences on social function in patients with schizophrenia-spectrum disorders. J Psychiatry Neurosci 2010;35:4954.

22. Dickerson F, Boronow JJ, Ringel N, Parente F. Social functioning and neurocognitive deficits in outpatients with schizophrenia: a 2-year follow-up. Schizophr Res 1999;37: 13-20.

23. Addington J, Penn D, Woods SW, Addington D, Perkins DO. Social functioning in individuals at clinical high risk for psychosis. Schizophr Res 2008;99:119-124.

24. Addington J, Addington D. Social and cognitive functioning in psychosis. Schizophr Res 2008;99:176-181.

25. Juckel G, Morosini PL. The new approach: psychosocial functioning as a necessary outcome criterion for therapeutic success in schizophrenia. Curr Opin Psychiatry 2008;21: 630-639. 
26. Helzer JE, Kraemer HC, Krueger RF, Wittchen HU. Dimensional approaches in diagnostic classification: Refining the research agenda for DSM-V. American Psychiatric Publishing Inc.;2008.

27. Kotov R, Chang SW, Fochtmann LJ, Mojtabai R, Carlson GA, Sedler MJ, et al. Schizophrenia in the internalizingexternalizing framework: a third dimension? Schizophr Bull 2011;37:1168-1178.

28. Boyette L, Swets M, Meijer C, Wouters L, Kahn RS,
Linszen DH, et al. Factor structure of the Yale-Brown Obsessive-Compulsive Scale (Y-BOCS) in a large sample of patients with schizophrenia or related disorders and comorbid obsessive-compulsive symptoms. Psychiatry Res 2011;186:409-413.

29. Kotov R, Ruggero CJ, Krueger RF, Watson D, Yuan Q, Zimmerman M. New dimensions in the quantitative classification of mental illness. Arch Gen Psychiatry 2011;68: 1003-1011. 